\title{
Empresas recuperadas: cooperación y conflicto en las nuevas formas de autogestión de los trabajadores
}

\author{
Bialakowsky, Alberto L.* \\ Robledo, Guillermo** \\ Grima, J osé Manuel*** \\ Rosendo, Ernestina y Costa, María Ignacia*** \\ * Director Proyecto UBACYT, Instituto de Investigaciones Gino Germani, Facultad de \\ Ciencias Sociales, Universidad de Buenos Aires. \\ ** Empresa recuperada IMPA (Industria Metalúrgica Plástica Argentina). \\ *** Integrantes del Proyecto UBACYT, Instituto de Investigaciones Gino Germani, \\ Facultad de Ciencias Sociales, Universidad de Buenos Aires. Agradecemos muy \\ especialmente la colaboración y los aportes de Horacio Campos (Empresa Recuperada \\ IMPA), Susana Presta y Natalia Benzaquén (cursantes avanzadas de las carreras de \\ antropología y sociología, respectivamente).
}

\section{Resumen}

El presente trabajo es el resultado de una investigación coproducida entre el equipo de la Universidad de Buenos Aires y los trabajadores de IMPA (Industria Metalúrgica Plástica Argentina), empresa recuperada por los trabajadores en el año 1998, después de ser llevada a una situación de quiebra por sus administradores. A fines de la década de los noventa, la crisis salarial, la crisis de legitimidad acerca de la propiedad privada conmovida por la crisis financiera, la flexibilización laboral y los procesos de desalojo social, han desencadenado y van gestando distintas formas de resistencia, entre las cuales se destaca un nuevo hecho socio-productivo, con antecedentes históricos escasamente homologables. El estudio se focaliza en el contexto del Movimiento Nacional de Empresas Recuperadas. Estas experiencias interrogan a los desarrollos de la sociología del trabajo al cuestionar la lógica de los procesos taylorista-fordistas-toyotistas y al enfrentar al desafío de construir teoría crítica y propositiva en acción coproductiva. En este sentido el artículo se dirige a analizar tres aspectos vinculados con las mutaciones en el mundo del trabajo: en primer lugar, el proceso social de trabajo a la luz de estas nuevas formas de gestión y autogestión que nacen de la resistencia de la clase trabajadora por preservar su reproducción como colectivo; en segundo lugar, la mutación interna del proceso social de trabajo en fábricas recuperadas y su encadenamiento con la producción de un proceso de trabajo social de creación institucional y cultural asentado en una b́gica de cooperación interinstitucional solidaria de carácter movimientista y, en tercer lugar, la emergencia de 
un cambio en la subjetividad de los trabajadores como resultado de la lucha frente a la dinámica de exclusión- extinción propia de las lógicas y materialidades neoliberales imperantes en la región a partir de las últimas tres décadas. El análisis de la temática propuesta implicará la utilización de una metodología investigativa cualitativa en el marco del paradigma de la complejidad y será abordado a partir de historias de vida, entrevistas, observaciones participantes y talleres coproductivos que darán cuenta, a través de las dimensiones de cooperación, poder y subjetividad, de las formas en que se recrean las identidades, la cultura y la conciencia colectiva-subjetiva en las empresas sociales.

Palabras clave: Autogestión, cultura laboral, trabajo, conocimiento empresarial, conflicto laboral.

\section{Recycling Companies: Cooperation and Conflict in New Forms of Worker Self Management}

\section{Abstract}

This paper is the result of research produced through colaboration between a group of researchers in the University of Buenas Aires and workers at the IMPA (Argentinia Metalurgic and Plastic Industry) a recycling company recovered and operated by workers in 1998, after having been led into bankruptcy by its administrators. At the end of the 1990s, the salary crisis, and the legitimacy crisis in the private sector due to financial crisis, labor flexibilization, and the process of social displacement, all combined and stimulated different forms of resistance, among others a new social-productive process with few similar historic antecedents. This study focuses on the context of the National Movement of Recovered Businesses (MNER). These experiences question the development of work sociology while doubting the logic of the Taylor-Ford-Toyota processes and challenging the development of critical pro-positive theory in co-productive activity. In this sense this article is directed towards analyzing three aspects related to mutations in the labor world; first, the social process of labor under these new forms of management and self -management which originate in the resistance of the working class in preserving its collective reproduction; second, the internal mutation in the social work process in recovered factories and the unchaining of the production of a social work process of institutional and cultural creation based on a logic of inter-institutional solidarity, cooperation of a work movement character; and third, the emergence of a change in the subjectivity of workers as a result of the fight against the dynamic of exclusion- the selfextinction of neo-liberal logic and materialism which have dominated the region over the last three decades. Tha analysis of this theme implies la utilization of a qualitative 
research methodology within the framework of the complexity paradigm, and is approached through life-histories, interviews, participant observation and co-productive workshops that reveal, through the dimensions of cooperation, power and subjectivity, the manner in which identities, culture and colective-subjective consciousness are created in social enterprises.

Key words: Self-management, labor culture, work, business knowledge, labor conflict.

Recibido: 03-11-19. Aceptado: 04-03-31

\section{I ntroducción}

El contexto argentino de la década del 90 ha estado signado por una profunda transformación de las relaciones laborales que rigieran hasta mediados de la década del 70, caracterizándose por procesos de flexibilización laboral, desregulación del mercado de trabajo y aumento significativo de la tasa de desocupación. La transformación del trabajador universal, propio del modelo fabril (taylorista-fordista), se orienta hacia la conformación de un nuevo tipo de trabajador, en el marco de una inversión de las reglas de regulación laboral y de la desmaterialización del colectivo como instrumento reivindicativo: "En este contexto las reglas contractuales, formales e informales, se alteran. Se produce un retroceso material del actor laboral frente al empresarial, que simultáneamente se acompaña de un debilitamiento de su base simbólica-referencial (...) Los cambios actuales introducen particularidades tanto a nivel micro como macro. Entre los aspectos micro, la visión del producto se remite a una visión individual, de oportunidades, de necesidades, de competencia entre pares. Desconoce entonces, el producto como resultado del colectivo (...)" (Bialakowsky y Rosendo, 1998: 13).

Sin embargo y pese a la vigencia de esas particularidades de las relaciones laborales forjadas principalmente en la década pasada, se produce un nuevo fenómeno creciente entre la finalización de los 90 y los inicios del 2000: “la toma o recuperación" de fábricas por parte de sus trabajadores. Si bien la existencia de cooperativas puede constituirse como un antecedente de este nuevo fenómeno, consideramos que los procesos actuales de recuperación poseen cierta especificidad sin precedentes que tornan fundamental su estudio y análisis.

En esta primera etapa de investigación de carácter exploratoria, nos hemos centrado en un enfoque de tipo cualitativo (I. Vasilachis de Gialdino, 1992), a través de la realización de entrevistas semi estructuradas a trabajadores de la fábrica IMPA, observaciones no 
participantes de los procesos de trabajo al interior de la fábrica y encuentros de coproducción investigativa entre trabajadores de IMPA y miembros del equipo de investigación UBACyT⒈

La selección de la fábrica IMPA como caso de estudio se funda en que lo consideramos como un caso paradigmático por su trayectoria y sostenimiento a lo largo del tiempo, dado que el fenómeno de las fábricas recuperadas comienza a adquirir relevancia luego de los sucesos de diciembre del 2001, y el caso IMPA se remonta al año 1998 continuando en la actualidad $\underline{2}$.

Si bien el proyecto de investigación se inscribe dentro de una problemática más amplia, cuyo objetivo general es comprender el fenómeno de las empresas recuperadas, en sus distintas variantes, en la coyuntura actual y en el escenario social futuro; este trabajo se propone centrar su atención en algunas dimensiones que consideramos remiten a la identidad y la cultura en las nuevas formas de gestión y autogestión de los trabajadores. En este sentido tomaremos en consideración las siguientes dimensiones: Movilización y conflicto; Proceso social de trabajo; Obreros o compañeros; El producto y Transmisión de conocimientos y difusión.

\section{Contexto y movimiento de empresas recuperadas}

Las movilizaciones del 19 y 20 de diciembre de 2001 marcaron un punto de inflexión en la profundización y multiplicación de movimientos político-sociales, sujetos a una diversidad de interpretaciones desde la resistencia a las prácticas de la multitud (Colectivo Situaciones, 2002; E. Cafassi, 2002; M. Hardt y A. Negri, 2002), que emergen en respuesta a la crítica coyuntura económica, política y social argentina. Estas nuevas formas de protesta (asambleas populares) y movimientos sociales de resistencia (piqueteros) convergen con la multiplicación de formas de gestión y autogestión de los trabajadores (micro emprendimientos, clubes del trueque, fábricas recuperadas) que no sólo expresan sus propios proyectos sino también el conflicto entre poder-sobre y antipoder (J. Holloway, 2002).

En particular, el avance del fenómeno de las fábricas recuperadas $\underline{3}$ puede visualizarse como un hecho social novedoso en el contexto socioeconómico argentino. En primer lugar, la movilización en torno a un icono hegemónico e ideológico, la propiedad privada, ha sido puesta en cuestión a partir de un proceso social que no solo comprende el vaciamiento de empresas y el consecuente desempleo, sino que también comprende la manipulación de la moneda a través de la aplicación primero de la Ley de Convertibilidad, luego el 
denominado "corralito financiero" con la consecuente incautación de fondos, la devaluación posterior, el proceso inflacionario que le sigue y el estallido de la actual crisis salarial. En segundo lugar, la incertidumbre política frente a las alianzas del poder político y económico y la succión del pago de intereses de la deuda externa. En tercer lugar, la nueva cuestión social que se ha agudizado a lo largo de los 90 caracterizada fundamentalmente por el desempleo y la pobreza crítica 4 que determina procesos de exclusión y extinción social. En este contexto las políticas sociales asistenciales, de carácter paliativo, no han logrado dar respuesta a los problemas que emergen de esta dinámica de acumulación y distribución $\underline{5}$. Y por último, en cuarto lugar, el mantenimiento de la estructura democrática que no logra impedir los extremos de la represión y el resguardo de los derechos sociales (Bialakowsky et al., 2002).

Si bien puede considerarse que las prácticas de autogestión han resurgido como un movimiento central a partir de diciembre del 2001 producto del clima social imperante descripto, cabe destacar que el desarrollo de estas experiencias se constituyó en estrategia de lucha contra el desempleo, desde fines de los 90. Por otra parte, estos procesos autogestivos, sin duda tienen antecedentes históricos, rupturas y continuidades dentro del movimiento de los trabajadores; en los 60 con el movimiento cooperativista, en los 70 con empresas autogestionadas y la participación empresaria de los trabajadores, como posteriormente la producción de una involución tanto en el movimiento autogestivo y cooperativo como del movimiento de los trabajadores reprimido por la dictadura militar. De ahí que el fenómeno de las fábricas recuperadas haya que analizarlo a partir de la cultura obrera acumulada y sus fracturas, irrupciones, como en su carácter novedoso y coyuntural en este particular contexto histórico (Bialakowsky et al., 2002).

En la evolución del fenómeno se destaca la conformación del Movimiento Nacional de Empresas Recuperadas integrado por las empresas recuperadas por sus trabajadores, que se autogestionan. O. Campos relata el surgimiento del movimiento: “(en el 2001) En ese momento no se pensó en un movimiento de empresas recuperadas, se pensó en ir a dar una mano ... íbamos a las marchas, veían el cartelito de IMPA, se acercaban y nos preguntaban si podían venir a conocer, y bueno eso fue de boca en boca, creciendo y los que tenían problemas iban a IMPA a preguntar como hacer, después vinieron de Entre Ríos, de distintos lados y ahí es donde se dijo, esto es a nivel nacional entonces vamos a hacer un movimiento. Ya Eduardo (Murúa), todas las semanas, estaba viajando de un lado a otro y bueno empezaron a haber reuniones aquí con 30 ó 40 personas de distintas empresas y empezamos a ver como nos comunicábamos, dejamos nuestro número de teléfono, cuando podía haber otra reunión hasta que por ahí dijeron que teníamos que hacer un movimiento con representación de todos y ahí surgió el Movimiento Nacional de 
Empresas Recuperadas ... y en esas reuniones que se hacen cada quince días, al principio era como que no había organización y decidimos elegir una comisión que nos represente y bueno eligieron a Eduardo (Murúa) y a (José) Abelli y en cada empresa recuperada creo que hay secretario o así... una comisión que representa en la empresa...".

En este marco el Movimiento Nacional de Empresas Recuperadas reivindica la ocupación de fábricas como una nueva forma de lucha contra la desocupación, el hambre y la pobreza. Sus principios son: 1. Asociación abierta y voluntaria; 2. control democrático por los trabajadores; 3. participación económica de los trabajadores; 4. autonomía e independencia; 5. educación, capacitación e información; 6. cooperación, integración y solidaridad entre las empresas y 7. preocupación e interacción por y con la comunidad. Entre sus principales objetivos pueden señalarse: reclamar por la modificación de la Ley de Quiebras트, propiciar la creación de un Fondo Fiduciario mixto, solicitar apoyo de los organismos de investigación para definir con el mayor nivel de rigor científico los indicadores de eficiencia acordes a las actividades que desarrollan las empresas del MNER, estudiar variantes a los sistemas previsionales vigentes (Reparto y Capitalización), conformar la Obra Social (Seguro Social) de los Trabajadores de las Empresas Recuperadas, entre otros $\underline{7}$.

En la actualidad, las disidencias ideológicas y políticas al interior del movimiento se cristalizan en la división entre el Movimiento Nacional de Empresas Recuperadas (MNER) y el Movimiento Nacional de Fábricas Recuperadas (MNFR). Estas dos organizaciones difieren en relación al tipo de organización y el futuro próximo de las empresas recuperadas, ya que mientras el MNER promueve la marcha de las fábricas bajo la modalidad de cooperativas y solicita el apoyo del Estado por medio de la creación de un fondo de capital inicial para la promoción de las mismas, el MNFR interpela al Estado para que se haga cargo de la deuda y la empresa pase a ser estatal pero bajo el control obrero (Contrera, 2003).

En esta última etapa, el movimiento de empresas recuperadas, ha buscado canalizar su proyecto político a través de su participación en los ámbitos de representación fundamentado en las palabras del presidente del MNER, Eduardo Murúa: “Nosotros siempre creímos que la lucha social tiene que estar reflejada en lo electoral ... No luchamos solamente por la recuperación de las fábricas sino por una sociedad distinta, por eso tenemos que reflejar la lucha social en la lucha política" (Murúa, 2003). No obstante esto, la elección de los partidos políticos a través de los cuales han elegido presentar candidaturas no se ha planteado de la misma manera o con iguales criterios en todas las 
empresas ocupadas $\underline{8}$.

\section{Movilización y conflicto}

La evolución del fenómeno de la recuperación de una empresa presenta dos momentos claves para comprender la reconversión del trabajador: la toma de conciencia por parte de los trabajadores acerca de las condiciones de trabajo y los peligros que deben enfrentar, y la participación en un proceso de resistencia e intensa movilización en pro de la conservación de la fuente de trabajo para su supervivencia. En palabras de un trabajador:

"Nosotros como viejos tomamos conciencia con la cooperativa hace cinco y pico de años cuando empezó el nuevo movimiento porque anteriormente nosotros no le dábamos ni cinco de bola a la cooperativa por eso lo que nos pasó: era obrero - patrón. No teníamos conocimiento de la parte interna de la plata y todas esas cosas, de lo que se vendía y no se vendía... Como nunca preguntábamos y nadie contestaba nada. A ellos les interesaba llevarse la guita, nosotros lo que menos pensábamos en la cooperativa. Ahora sí. [...] Lo que nos surgió es que estuvimos tres meses parados pidiendo plata, pidiendo para comer. Entonces tenés que tomar conciencia de tu trabajo y de salir todo adelante" (Relato de un trabajador de la fábrica IMPA, Encuentro de coproducción investigativa, 2003).

Este proceso de identificación del conflicto y de lucha renueva la identidad del trabajador, autotransformándolo y diferenciándolo de aquellos trabajadores ajenos a las etapas de ocupación y puesta en marcha del proyecto de autogestión por su incorporación en la fábrica en etapas posteriores. En otras palabras, el trabajador, en la lucha, adquiere una identidad social que le permite hablar de un "nosotros" construido a partir de la inclusión y confirmación de sus miembros en un proyecto común (Bauman, 2002 y Sennett, 2000).

El conflicto que atravesaron los trabajadores de IMPA, tiene sus raíces en el modo en que la relación capital-trabajo fue desenvolviéndose a lo largo de la historia y de la propia constitución de la clase obrera como clase para sí. En otras palabras, la posibilidad de identificación de un conflicto vivido subjetiva y colectivamente, se convierte en un principio de explicación social e histórico (Touraine, 1992).

Es importante analizar cómo "la insatisfacción personal, el sentimiento colectivo de noparticipación y la conciencia de alienación" (Touraine, 1992: 107) se reúnen de manera tal que dan lugar a prácticas que se gestan en el contexto de situaciones de extrema conflictividad y que, en el caso de las fábricas recuperadas, tienden a generar una nueva 
conciencia basada en prácticas y percepciones que incluyen al colectivo de trabajo como fundamento de su existencia y desarrollo.

Desde nuestra perspectiva, la puesta en marcha de la producción enfrenta desafíos que involucran a un colectivo de trabajo más amplio y heterogéneo, la redefinición de las antiguas formas de cooperación y de organización del proceso productivo. Este proceso de recreación del trabajo se asienta sobre tensiones entre el conocimiento acumulado, la crítica a los procesos tradicionales y la necesidad de consensuar prácticas y saberes con vistas a la creación de un nuevo orden. Proceso que no se da en forma lineal, unidireccional sino de manera móvil, compleja y conflictiva.

Las transformaciones de la "técnica de trabajo" 9 tienen fuertes implicancias tanto para el sujeto como para el colectivo. La tradición de un acto técnico significa el poder ser comprendido por los demás, poder reproducirse, rutinizarse y básicamente, transmitirse. En este sentido, la técnica hace a la identidad de cada uno de los trabajadores, pero también a la pertenencia a un grupo o colectivo: "Y de todas maneras es necesario para que los otros reconozcan la validez de ese hacer, que comprendan su sentido, es decir que el acto esté situado en relación con una tradición común. Finalmente, el reconocimiento de la calidad de ese vínculo que se establece por el acto entre ego y lo real supone que este acto no sea absurdo sino eficaz. Esta dinámica de la identidad permite captar cómo la técnica moviliza siempre apuestas de identidad, y recíprocamente, cómo la conquista de la identidad y la búsqueda de reconocimiento de los otros implica siempre una relación con lo real mediatizada por una técnica" (Dejours, 1998: 201).

La ruptura o transformación de las técnicas de trabajo supone entonces transformaciones no sólo en lo que respecta al 'hacer' sino también en las relaciones del colectivo de trabajo. La vigencia de una técnica (tradicional) implica la existencia de reglas de trabajo comunes, sobre las que se asienta el reconocimiento y la pertenencia a un colectivo. Por lo tanto, la transformación de las técnicas tradicionales requerirá de un complejo proceso de reinstauración de técnicas (y reglas de trabajo) comunes para que el grupo pueda seguir produciendo, y para que el sujeto se sienta reconocido e incluido en el colectivo. Probablemente la movilización y la lucha se hayan constituido en las bases sobre las que se erigieron las nuevas reglas y técnicas de trabajo que hacen que el colectivo pueda seguir funcionando.

Entre las distintas fases que atraviesa la recuperación de las empresas por parte de los trabajadores, la etapa productiva evidencia diferentes conflictos que desnudan los aspectos sociales del proceso de trabajo. El poder no desaparece sino que se reintroduce 
en diferentes formas. Es cierto que hay un desplazamiento del conflicto principal empleador-trabajador, pero emergen nuevas formas de apropiación del capital simbólico (Bourdieu, 1988), tales como haber participado o no de las gestas fundadoras que exigieron a los trabajadores riesgos, sacrificios e inversiones personales y familiares. Podríamos suponer entonces que, en mayor o menor medida, es dicho capital simbólico el que está en juego al interior de la fábrica, pero también, el que se halla en la base de las relaciones que los trabajadores de IMPA construyen con la sociedad, con otras instituciones y movimientos populares.

Estas acumulaciones crean una distinción entre los fundadores, los históricos, y aquellos otros nuevos trabajadores que sin desconocer este pasado no poseen la vivencia de las gestas fundadoras. En este sentido, transformaciones culturales, que representan formas identitarias y cosmogonías laborales, revelan posicionamientos conflictivos susceptibles de generar fuentes de poder al interior de la fábrica. Sin embargo, podemos suponer que estas posiciones no serán estables ni fijas sino que podrán ser modificadas por los sujetos en la medida que luchen para acrecentar su capital simbólico según las reglas tácitas del juego (Gutiérrez, 1994). Asimismo, podemos considerar que el espacio social está arraigado tanto en las estructuras espaciales como en la subjetividad, entonces, el espacio será un lugar para la afirmación y ejercicio del poder (Bourdieu, 1999). Por tanto, aquellos trabajadores que participaron en la lucha de recuperación de la fábrica y que cuentan con largas trayectorias de trabajo, serán quienes ocupen posiciones de mayor reconocimiento.

“Cuando se escuchó el ruido de las máquinas trabajando... Era algo fuerte, una alegría enorme. Una cosa es contarlo y la otra es sentir esa alegría. Los primeros tiempos fue duro. Llevábamos 5 pesos por día y 50 pesos los viernes. Y había veces que teníamos que dejar algo de los 50 pesos para la materia prima. Empezaron a venir compañeros que se habían ido porque habían conseguido otros empleos, pero empezaron a venir y a preguntar si había un lugarcito. Eso generó un conflicto entre los que participaron en la lucha y los que no... A medida que facturáramos íbamos a mejorar y de 5 pesos pasamos a 10 y de 50 a 70, 80 y cuándo llegamos a 100! El ánimo de la gente [luego de la movilización] cambió en un 100\%. Imagínate si te pagaban 2 pesos cuando te pagaban. Ahora no hay patrón, no hay dueño, somos todos interesados!" (Relato de un trabajador de la fábrica IMPA, Encuentro de coproducción investigativa, 2003).

“La relación con los nuevos es de compañerismo, de trabajo, porque no te puedo decir tanto de familia porque no, pero... Claro que ellos vinieron con la cama hecha, ya todo está hecho, nada más que a veces si se los precisa van a tener que ir a la movilización igual" (Relato de un trabajador de la fábrica IMPA, Encuentro de coproducción 
investigativa, 2003).

A su vez estas distinciones se materializan en las formas de contratación y en los salarios que perciben los trabajadores recientemente incorporados. De esta manera las condiciones laborales en las que estos trabajadores se inscriben dejan traslucir una nueva tensión entre el impulso de prácticas cooperativas y la implementación de prácticas flexibilizadoras típicas de las relaciones laborales actuales. En palabras de los trabajadores:

“... Lo que pasa es que cuando vienen, no saben el trabajo que tienen que hacer. Tienen que pagar derecho de piso hasta que aprenden, entonces a los tres meses si tienen buen comportamiento y aprenden, ya prácticamente quedan asociados.... [...] I gualmente va a haber diferencias de sueldo porque no van a cobrar lo mismo que cobramos nosotros. Va a haber una diferencia de... ponele de 30 pesos, 40 pesos, de acá a un cierto tiempo" (Relato de un trabajador de la fábrica IMPA, Encuentro de coproducción investigativa, 2003).

De este relato se infiere que la diferencia salarial actúa como principio modulador de una parte del conflicto entre antiguos y nuevos trabajadores.

\section{Proceso social de trabajo}

El establecimiento de un sistema productivo requiere un determinado orden, previsibilidad, ajustes debajo de los cuales se desenvuelve la cooperación social como motor del proceso productivo, es el colectivo el que funda la posibilidad de la producción. El colectivo - a través de la cooperación- es un bien distinguible y en el que podríamos afirmar se basó centralmente el desenvolvimiento del capitalismo, tanto en su aspecto de plan de producción como en su aspecto de dominación y disciplinamiento de los trabajadores (Marx, 1988b). Al mismo tiempo podríamos situar en este punto la capacidad esencial del dominio sobre el hombre y la materia, y por ende, los conflictos que emergen de la explotación de la fuerza productiva y de la naturaleza. De ahí que todo plan, posee un diseño (no visible) de rediseño del hombre trabajador, del colectivo, la máquina y los otros bienes conforman en su silencio una posibilidad maquínica más allá de la máquina misma (Gaudemar, 1978).

Los sistemas de autogestión bajo formas cooperativas plantean un rediseño de los procesos productivos y de las articulaciones laborales al interior de las empresas. Se introducen una serie de elementos vinculados con: a) la participación; b) la disolución de 
las relaciones jerárquicas y las nuevas formas de cooperación; c) la disciplina, el control, la autonomía y el consenso en el proceso social de trabajo; d) la rotación de tareas.

En cuanto al primer punto, la participación de los trabajadores en las asambleas señala un reposicionamiento de los mismos respecto del proceso de toma de decisiones concernientes a los intereses del colectivo.

“Uno puede participar en decisiones. Obviamente que hay gente que se encarga de ver cómo es el trato con los proveedores, porque nosotros no entendemos mucho de eso, pero si de repente a nosotros se nos ocurre una idea nueva para poder cobrarle a la gente que se le vende...., en cierta forma, a la gente que vos le vendes o al que le comprás: 'Mirá te pago más adelante porque ahora no puedo', si a nosotros se nos ocurre algo, por ejemplo, una idea mejor, el Consejo las evalúa y si es buena la toman" (Relato de un trabajador de la fábrica IMPA, Encuentro de coproducción investigativa, 2003).

En el proceso de trabajo también se refleja una mayor participación del trabajador al poder introducir iniciativas propias, salir de su función específica y emprender un proceso activo que facilite la interconexión con las diferentes áreas agilizando simultáneamente el sistema de comunicación y de producción.

“Nosotros, por ejemplo, si vemos que no tenemos materia prima para fundir, estamos sobre los de arriba [refiri éndose a los administrativos], constantemente. No nos quedamos esperando que cuando a ellos se les de la gana de comprar. Ahora no! Si no tenemos materia para fundir, vamos directamente a hablar con él para que las cosas se hagan enseguida. Los compañeros preguntan: che, qué pasa que no tenemos materia para fundir y nosotros que estamos más o menos al frente tenemos que ir..." (Relato de un trabajador-coordinador de la fábrica IMPA, Encuentro de coproducción investigativa, 2003).

En cuanto al segundo punto, la disolución de las relaciones jerárquicas y las nuevas formas de cooperación, es necesario resaltar las diferencias entre los sistemas de imposición, donde se establece la cooperación despótica y donde la subjetividad del trabajador se produce entre la dialéctica de la adaptación y la resistencia, y los sistemas de autogestión donde si bien persiste una organización jerárquica dentro de la fábrica (coordinadores y trabajadores), ésta tiende a disolverse bajo las nuevas formas de cooperación y solidaridad entre co-trabajadores. Se puede señalar al respecto que los trabajadores viven intentando y creando formas de cooperación no despótica o consensuada. Es un intento renovado cada dáa a través del método de imponer una 
racionalidad distinta. Intento en el que se juega la subjetividad en sus niveles, singulares y sociales.

“El grupo de nosotros está todo junto, está trabajando todo junto, lo que le llega a uno, le llega al otro lo mismo" (Relato de un trabajador-coordinador de la fábrica IMPA, Encuentro de coproducción investigativa, 2003).

“Acá somos todos compañeros, trabajando como todos los compañeros. Yo soy un capataz, un coordinador de la sección pero yo si tengo que trabajar a la parte de ellos, hago lo de ellos, no tengo ningún problema, somos todos iguales. Acá no se los manda a las personas así directamente, así nomás, se trata la forma de mandarlos para que sean todos unidos, que la persona comprenda que tiene que hacerlo... el compañerismo de todos" (Relato de un trabajador-coordinador de la fábrica IMPA, Encuentro de coproducción investigativa, 2003).

En el caso de la autogestión, al referirnos a un sistema autosostenido, el desafío es alimentar la cooperación, el trabajo colectivo, desde lo subjetivo. En el discurso se apela al esfuerzo individual de cada trabajador como un aporte indispensable para la prosperidad colectiva. Esto induce no sólo a comprometerse subjetivamente con el trabajo sino también a mantener un determinado orden al interior de la fábrica.

"Si todos no colaboramos, nos quedamos estancados" (Relato de un trabajador de la fábrica IMPA, Encuentro de coproducción investigativa, 2003).

En tercer lugar, el disciplinamiento expresado como forma de dominación y control patronal para el funcionamiento del trabajo colectivo es resignificado en la medida que las relaciones tienden a horizontalizarse. Se juega aquí la cuestión de la jerarquía que se impone al parecer de manera necesaria en todo proceso. La misma es a la vez arquitectura de sometimientos y arquitectura de emergencias (Morín, 1998). Por ello se puede señalar que, la disciplina aplicada a la fuerza de trabajo a través de la máquina (disciplina maquínica) representa un doble proceso de objetivación e interiorización de la disciplina (J. P. De Gaudemar, 1978) del cual los trabajadores autogestionados no están exentos y se encuentran en permanente conflicto promoviendo, paradójicamente, el consenso y luego la creatividad como respuesta al ejercicio del biopoder fabril.

“Trabajador: No por ser autogestionada todo es tan maravilla, hay veces que hay ciertas personas que hay que marcarles ciertas cosas que se tienen que respetar. Si el resto de los asociados, de los compañeros, las respetan... 
MI: ¿Lo marcan Ustedes o lo hace el coordinador?

Trabajador: Entre nosotros, claro" (Relato de un trabajador de la fábrica IMPA, Encuentro de coproducción investigativa, 2003).

“Acá nadie tiene presión, todos sabemos lo que tenemos que hacer, nadie tiene presión sobre su trabajo. Sabes tu producción diaria, las 9hs. Nadie anda por detrás de vos, mirándote, siguiéndote. Creo que tenés más libertad y trabajas mejor, estás tranquilo" (Entrevista a un trabajador de la fábrica IMPA, 2003).

En cuarto y último lugar, la división social del trabajo, la especialización y rutinización de tareas perduran en la organización del trabajo de fábricas autogestionadas, sin embargo los trabajadores comienzan a asumir nuevos roles y un perfil más flexible y polivalente (Palomino, 2003) que en esta situación se propone como factor desalienante. En palabras de un trabajador administrativo: "todos están aprendiendo a hacer todas las tareas".

\section{Obreros o compañeros}

"S: El otro día, cuando hablamos, usted me había dicho, cuando yo le dije "obreros", me dijo "no, compañeros".

Trabajador 1: Acá se trata de compañeros, ya no se trata de obreros.

Trabajador 2: Es completamente distinto, vos no vas a comparar, aquí somos todos compañeros como dijo Carrizo. Aquí antes había un patrón por detrás tuyo, es completamente distinto, por más bueno que sea, el patrón siempre va tratar de que le produzcas más, de que le tenga prácticamente en línea, aquí cada uno sabe lo que va a hacer, lo hace lo mejor que se puede y nadie anda por atrás apurándolo, eso es la diferencia" (Trabajadores de la fábrica IMPA, Encuentro de coproducción investigativa, 2003).

Del relato se desprenden algunas consideraciones referidas a la cuestión de la identidad que moldea el proceso de lucha y la implementación de prácticas autogestivas, las cuales se asientan en el desplazamiento de la categoría "obrero" y en una reapropiación del término "compañero" como la manera de reconocerse y de reconocer al otro. Esto nos conduce a reflexionar sobre tres aspectos vinculados con: a) las representaciones simbólicas e imaginarias que constituyen las articulaciones laborales (Bialakowsky y 
Fernández, 1994); b) la utilización de categorías y nominaciones propias del campo político-ideológico para designar a los sujetos que participan del mundo fabril y que han vivido experiencias de lucha colectiva y c) la nueva cultura laboral producto de los cambios operados en el proceso social de trabajo que bajo formas cooperativas disuelve las jerarquías y las formas de cooperación típicas.

Tenemos entonces tres conceptos fundamentales que adquieren una particular definición en el marco de las empresas recuperadas, ellos son: cooperación, confianza y reconocimiento. La cooperación supone más que la coordinación lógica y cognitiva exitosa en las actividades; supone también la voluntad de los trabajadores para trabajar juntos: sin cooperación no sería posible la producción (Dejours, 1998). La cooperación implica entonces relaciones de confianza, tanto entre colegas como con sus coordinadores, estructura que hace posible la instauración de acuerdos y normas que regulen la forma de ejecutar el trabajo.

En este sentido, si bien las empresas recuperadas proponen una disolución de las jerarquías (gerencia-capataces-obreros), no quedan exentas de una regulación normativa que articule las relaciones entre trabajadores y la realización del trabajo dentro de una previsión productiva. El carácter colectivo y social del trabajo se despoja del velo del capital para adquirir un sentido renovado para el trabajador. Su redescubrimiento da lugar a nuevas formas de concebir y actuar en relación con el trabajo y en la construcción de subjetividad y de vínculos sociales con los demás co-trabajadores.

Esta nueva cooperación se funda en la confianza y en la voluntad de los trabajadores, pero también en el reconocimiento sobre el hacer lo cual supone una ganancia para la identidad del trabajador (Dejours, 1998). Este reconocimiento necesario en cada organización de trabajo implica, desde nuestra perspectiva, una acumulación identitaria que resulta incluso previa a la puesta en marcha de la organización productiva en el caso de las empresas recuperadas. Punto que en alguna medida establece una diferenciación entre los participantes activos del proceso de "toma de la empresa" y los nuevos trabajadores que se incorporan a la organización de trabajo sin haber atravesado ese momento fundante del colectivo.

Entonces, si bien la desvinculación del capital del proceso de producción impacta en la subjetividad del trabajador reconvirtiéndolo, no por ello diluye otros posicionamientos conflictivos que subyacen a las relaciones laborales (nuevos trabajadores y viejos trabajadores; trabajadores movilizados y no movilizados). En este sentido el concepto de compañeros no disuelve la conflictividad latente. 
Desde esta perspectiva, podemos hablar de co-productores, del significado de producir juntos, bajo un tipo de racionalidad y sentido que a la vez reconoce el capitalista, produce la resistencia del trabajador; la autogestión bajo el modelo de autogestión puede ser pensada como construcción y creación de nuevos consensos aunque, como hemos visto, no exenta de conflictividad.

\section{El producto}

La mercancía es un producto destinado desde el principio a la venta y al mercado. El carácter fetichista de la mercancía se origina en "la peculiar índole social del trabajo que produce mercancías" (Marx, 1988c: 89), es decir, en las relaciones sociales de explotación que subyacen a la creación y el intercambio de los productos. Desde esta perspectiva, el desafío que enfrentan las fábricas recuperadas y autogestionadas es mostrar que el producto no es resultado de la explotación humana en un doble sentido: por el proceso mismo del trabajo (intensidad y dominación psicofísica) y por el producto del trabajo mismo, sino que la calidad del producto y su utilidad social se deben a un proceso más liberado, de producción consensuada.

"La diferencia pasada a la actual es que en el pasado no le importaba a la gente cómo salía [el producto] porque lo hacía para otro y actualmente uno lo hace con más ganas, con más atención y más esmero para que el material no vuelva" (Relato de un trabajador de la fábrica IMPA, Encuentro de coproducción investigativa, 2003).

“Y producir lo más posible. Es más, a veces producimos más de lo que pide él [refiriéndose al coordinador]. A veces hacemos en doce horas laborando cinco coladas y yo quiero hacer seis, si llegamos a hacer las seis, las hacemos" (Relato de un trabajador de la fábrica IMPA, Encuentro de coproducción investigativa, 2003).

Por otra parte el control de la calidad del producto y la intensidad del trabajo, a efectos de que se acreciente la productividad (Marx, 1988a), deja de estar a cargo del capitalista para constituirse en un proceso autosostenido que depende del esfuerzo del colectivo. Sin embargo, la estructura de comercialización y la obtención de dinero para la sobrevivencia en las empresas recuperadas continúa subordinada a la dimensión abstracta del producto en tanto su finalidad básica es la creación de valores de cambio.

“Trabajador A: Nosotros trabajamos en conjunto, no contamos con ingenieros, con nada. Nosotros hasta ahora confiamos en nosotros, si dentro de cinco años la confianza se 
pierde, vendrá otro. Pero hasta ahora nos la bancamos nosotros, así como levantamos la fábrica solos, tenemos que seguir continuando así.

Trabajador B: No por el hecho que no entre nadie de afuera.

Trabajador A: Pero un ingeniero que tiene un estudio no te va a llevar los mil pesos por mes, entre tres mil y cuatro mil pesos, te va a pedir para venir cinco o seis horas

Trabajador B: Tomar a alguien especializado sería en última instancia" (Relato de un trabajador-coordinador de la fábrica IMPA, Encuentro de coproducción investigativa, 2003).

“El control de calidad es el maquinista que trabaja en la máquina, es él el que sabe y el que no sabe" (Relato de un trabajador-coordinador de la fábrica IMPA, Encuentro de coproducción investigativa, 2003).

En síntesis, el proceso de creación de mercancías para el mercado implica un doble desgaste: a) de los materiales, de las máquinas y el ambiente y b) del propio trabajador que consume su energía y su vida en la fábrica. El capitalismo demanda ambos procesos para obtener plusvalía y el trabajador así los padece ante su necesidad vital del salario. Sin embargo, en las fábricas autogestionadas, estas dimensiones de alienación se enfrentan al desafío de un rediseño acerca de la intensidad de los tiempos de producción, la creación de hábitos de seguridad, de detección de riesgos, de calidad del producto y de su relación con el consumidor y el ambiente.

\section{Transmisión de conocimientos y difusión}

Un rasgo fundamental de la recuperación de fábricas nos remite al proceso que atraviesan los trabajadores en el cual adquieren conciencia crítica. En este sentido, no sólo se produce una renovación de la identidad del trabajador sino que además se emprenden otros desafíos vinculados con la acumulación y distribución de conocimiento (Bialakowsky y Fernández, 1994).

En cuanto a la primera dimensión, entendemos que el diseño productivo y las nuevas tecnologías son parte del proceso de producción. La pregunta que se formula es cómo se enfrentará este dilema tecnológico acerca de la productividad. En diálogo con los trabajadores: 
“Trabajador: Yo te voy a decir: ideas tenemos muchas, pero nos falta plata. Nosotros tenemos la idea de modificar la parte de fundición, la parte de esto, o sea, pero hoy en día es mucha plata...

MI: ¿Modificarlos con qué idea?

Trabajador: Para mejor la producción. Por ejemplo una lingotera que, prácticamente, funda 150 kilos por placa, entonces vos rendís más, pero falta esto [dinero]. Como está la situación hay que hacer una modificación muy grande.

MI : ¿Y cómo piensan que se puede dar este salto?

Trabajador: Eso tendría que ser una maquinaria más moderna, las máquinas nuestras ya no servirían. Sirven para el trabajo que estamos haciendo nosotros, pero para hacer una producción más grande tenés que tener una máquina de ...

MI: Piensan en la posibilidad de articular con otras instancias, por ejemplo, con instancias de gobierno, otras empresas, otras IMPA, para poder acceder al capital necesario?

Trabajador: $Y$ eso tendría que ser un préstamo muy grande del gobierno para poder modificar..." (Relato de un trabajador-coordinador de la fábrica IMPA, Encuentro de coproducción investigativa, 2003).

En cuanto a la segunda dimensión, podemos señalar dos pasajes: a) El pasaje del conocimiento individual al colectivo: "La puesta en colectivo implica una doble transformación del saber individual en una materia socializable y por lo tanto constructiva en sus dos sentidos, en el reconocimiento sobre la nueva identidad del trabajador no asalariado y la legitimidad de su práctica, y complementariamente la porción de poder que acompaña reapropiarse de sus instrumentos específicos del conocimiento técnico, para resolver finalmente en unidad la producción y la autoproducción" (Bialakowsky et al., 2003) y b) La lucha política colectiva que trasciende las puertas de la fábrica y redefine la relación entre el afuera y el adentro. Lo que se descubre es la capacidad de crear un método de traducción del conflicto privado, de un grupo de trabajadores con la patronal, en un tema público, en la lucha por la reivindicación de derechos sociales (Bauman, 2001).

“Como dicen ustedes, tienen la oportunidad de estar en una fábrica pero si no entran, 
cómo van a saber. Eso es lo que pasa a mucha gente. Por eso yo recalco que a los chicos del colegio o de la universidad o primaria, este, esto es una novedad y es muy lindo que lo vayan conociendo, pero la sociedad afuera, si no entran a una fábrica, ignora lo que nosotros hacemos, y hay gente grande que viene y ven el pomito, ven los tejos que se hacen y dicen: ¿y esto de qué sale? A mí me pasó que vinieron, este, y uno me pidió un pedacito de papel para llevar y poner en la... y un pomo. El papel lo llevó de ahí de IMPA, que dice IMPA que lo iba a pegar para ponerlo en la oficina. Después lo llevé a pomos y vieron, no querían creer que del tejo ese salía el pomo, y me pidió un pomito chico para llevarlo. Cosa que no... desconoce la sociedad. A veces peleamos nosotros aquí adentro por defender la fuente de trabajo, bueno, la gente comprende pero no ve el movimiento que hay, esto es como un hormiguero, donde todos hacemos, ponemos un granito de arena, uno solo no hace nada adentro de una fábrica, tiene que ser el conjunto que trabaje tanto la que hace la ayuda como la que organiza" (Relato de un trabajadorcoordinador de la fábrica IMPA, Encuentro de coproducción investigativa, 2003).

“S: Y con relación a la lucha ¿usted cree que esa lucha quedó solamente en lo que es IMPA o se traslado a la sociedad?

E: No, se trasladó a la sociedad. Ahora hay muchas cooperativas. Antes se conocía casi a IMPA nomás. No sé si sabrá usted, el presidente es Murúa, de las cooperativas recuperadas" (Entrevista a un trabajador de la fábrica IMPA, Encuentro de coproducción investigativa, 2003).

De esta manera, la materialización de la cultura laboral y la sustentabilidad del cotrabajo radican en la posibilidad de crear nuevos conocimientos y de distribuir los acumulados, traspasando los muros de la fábrica. Se descubre entonces el saber del colectivo y la posibilidad de diálogo con otros actores sociales tales como los consumidores.

Pero el co-trabajo no sólo recrea el saber técnico en relación a la producción sino que, como hemos visto a lo largo del artículo la ponencia, también recrea las relaciones entre trabajadores, su vínculo con la organización del trabajo y la conformación del colectivo y del nuevo proceso de cooperación. En síntesis, una nueva y singular propuesta de proceso social de trabajo.

\section{Reflexiones a modo de conclusión}

Diversos autores e intelectuales se han visto interpelados por la nuevas experiencias 
autogestivas de los trabajadores; por nuestra parte podríamos señalar algunas peculiaridades sobre los aportes de este trabajo. La primera refiere a tomar dimensiones claves en la reconstrucción del proceso identitario y los elementos relevantes para la construcción de una cultura laboral alternativa; la segunda peculiaridad, si se nos permite, refiere a nuestro enfoque metodológico que intenta ir más allá de la elaboración etnográfica y a una profundización de la IAP (investigación acción participativa) ensayando la coproducción investigativa, isomórfica -en este caso- a la producción cooperativa que intentamos analizar, metodología que incorpora la interpretación compartida en el flujo productivo del conocimiento.

Este objeto de análisis contiene elementos muy complejos a desarrollar tanto por la naturaleza de las dimensiones de identidad, cultura y conciencia como por la forma innovadora de la experiencia. Podría afirmarse que la mayońa de los recursos conceptuales de la sociología, la economía y la psicodinámica del trabajo provienen del reconocimiento o bien la subyacencia del conflicto permanente entre la fuerza del capital y la fuerza del trabajo. Las experiencias cooperativas de las empresas recuperadas remiten a otro escenario donde el conflicto principal ha sido desplazado en la confrontación con las fuerzas institucionales externas del poder judicial y político, del mercado y de los proveedores.

En este escenario no exento de conflictos (auto)observamos rasgos relevantes en la construcción de nuevos "para sí" que podrían partir de una serie de tensiones que pasamos a resumir.

En primer lugar conviene señalar que en esta producción -como en la historia natural del capitalismo- se juega la capacidad de articular el colectivo, darle sustancia material, convertirlo en un instrumento de producción. Este instrumento de producción conceptualmente puede remitirnos a dos fuentes de comprensión clásicas, ya sea en Marx sobre el capítulo de la cooperación ya sea en Owen en el montaje de una organización cooperativa. En la posesión del colectivo y la distribución del poder social tiene lugar una disputa que en el capitalismo se resuelve con la instrumentación de la cooperación despótica, con aquel plan que con apariencia de neutralidad controla férreamente el proceso de trabajo y exige el disciplinamiento del trabajador a la cadena de montaje. I gualmente el despotismo no es una imposición totalmente externa del método; exige una aceptación subjetiva, o con otras palabras una auto-des-subjetivización permanente, un proceso inagotable de extra ñamiento subjetivo.

En el proceso de trabajo asalariado, que precedió a este escenario cooperativo, el 
colectivo subyace oculto por la dominación, las normas de producción, los reglamentos, los controles de calidad, la distribución de las máquinas y los tiempos. La producción capitalista depende vitalmente del trabajo colectivo-cooperativo pero hace del control y de la necesidad del asalariado su instrumento principal de coerción; la cooperación sustancial del colectivo se encuentra en este proceso naturalizada, oculta en el trabajo mercancía, más podríamos afirmar que el plusvalor depende de este plus cooperativo colectivosubjetivo. El problema con que se enfrenta la producción cooperativa, en el sentido no salarial, es la articulación-materialización del colectivo con la acentuación del consenso y la reducción del control, la vigilancia y la sanción. El proceso de subjetivación lleva la resistencia subjetiva-colectiva a planos distintos del conflicto tradicional fabril y pone en evidencia permanente la construcción y distribución del poder.

Este desplazamiento - aunque no eliminación del conflicto interno- pone en evidencia una cuestión también naturalizada y poco comprendida; la unidad en el proceso de trabajo entre subjetividad y sociabilidad, entre la construcción de la singularidad y la proyección del ser social. La tarea en el puesto de trabajo no es una simple tarea sino la configuración del mundo, en sentido clásico el mundo de los modos de producción y la producción del ser genérico; aquí, ahora la representación de lo macro en lo micro y la proyección de lo micro en lo macro, la re-unión de la fragmentación subjetiva-escisiónimplícita en la dominación. De ahí que el planteo sobre el proceso de trabajo productivo tiene el carácter explícito de proceso social de trabajo al interior y de proceso de trabajo social hacia el exterior. La sustentabilidad depende entonces también de la dimensión política del quehacer cotidiano.

Cabe ahora afrontar discursivamente tensiones sobre las cuales se apoyan algunos fundamentos y representaciones de la propuesta de "recuperación de empresas". Una cuestión fundante es la revisión de la identidad asalariada y la emergencia -con otros contenidos- de la categoría "compañeros". Este anclaje identificatorio y cultural, conciencia nueva, mutación de sí para sí, implica una nueva legitimidad, dignidad del trabajador y forma organizativa al mismo tiempo. La categoría laboral de compañero instituye una forma horizontal de concebir la producción, que en lo observado rompe con el taylorismo secular, y aún con el toyotismo adaptativo, en la medida que la propuesta horizontaliza la decisión, el control y la construcción del colectivo. El atributo que descubrimos revela el carácter coproductor donde la relación de dependencia se troca por la relación coproductiva, este proceso de subjetivación no elude la tensión de la dominación que puede aparecer por ejemplo en los rituales de iniciación entre históricos y nuevos trabajadores co-operantes. 
Ahora bien, entre las tensiones que surgen en la nueva gesti ón coproductiva debemos no perder de vista la problemática referente a las nuevas condiciones de trabajo. En este sentido, el eje del conflicto se desplaza en la medida que ante la necesidad de producir, se asumen nuevos riesgos y mayor desgaste por el empobrecimiento de las condiciones de trabajo y los medios de protección del trabajador. El padecimiento antes instrumentado por el ejercicio del poder gerencial se torna ahora en una problemática al interior del proceso productivo, determinado -o autodiseñado- por la tecnología anterior que desgastaba hombres y naturaleza, sin pausa y sin medida. Desde esta perspectiva, debería haber un rediseño tecnológico que contemplara estos dos cuidados.

Otra cuestión, sin duda relevante, es el producto y sus representaciones, el producto como mercancía, clásicamente contiene formas fetichistas, incluso podríamos afirmar ahora totémicas; estas formas discursivas inherentes al producto mercancía por un lado cosificaban el trabajo productor precedente, por el otro ocultaban las formas de explotación y desgaste obrero que la sustentaban. El desafío consiste no sólo en variar las formas de dominación productiva, el plan productivo como ejercicio despótico, sino proyectar hacia la sociedad, hacia el usuario y el consumidor, otras formas representacionales acerca de la calidad productiva desfetichizada. Se trata de otra valoración que los iniciados en el tercer sector, la economía -política- solidaria, han comenzado a revalorizar. La economía entonces se dirige al intercambio entre productores no sólo entre productos equivalentes en su valor monetario que ocultan sus formas productivas. La revalorización enfrentará seguramente la competencia capitalista con dificultad, probablemente con conflicto en esta zona de representaciones sociales y productos provenientes del mercado.

Producto y proceso productivo se descubren en este proceso no escindidos, lo cual requiere una recuperación del todo en la parte, y de la parte en el todo. Este requerimiento se traduce en una interrogación de cómo formar parte del mundo al borde de ser expulsados. Resulta un momento clave para interrogar sobre los encadenamientos causales que conducen a la inermidad del trabajador. La inermidad tiene muchos significados subjetivos, colectivos y sociales. A partir de esta encrucijada se descubren cadenas causales entre las formas de producir productos y producir sociedad. De ahí que el saber -aun el técnico - resulta un saber no neutral, no adiafórico (moralmente inocuo) en el sentido que lo expresa Bauman. El saber es necesario, entonces, para encontrar armonía entre el despliegue del proceso productivo y su desgaste laboral, social y natural. Puede ser así que en la caída del carácter velado del trabajo-mercancía se descubra no sólo el biopoder fabril, sino el equívoco dominio antropocéntrico - del desgaste sin retornode la naturaleza. 
Podríamos avanzar sobre otros aspectos igualmente relevantes; sin embargo, resulta prudente detenernos sobre este haz apretado que contiene algunos cabos de la identidad, la cultura y la conciencia subjetiva, para afirmar como ensayo que la prueba que enfrentamos será reconstruir la lucha laboral con estos otros desplazamientos del conflicto que requieren ahora nuevos imaginarios y nuevas lógicas, más versátiles, menos maniqueas y más dialécticas. Para ello destacamos como herramienta el pensamiento colectivo, siempre subyacente, pero oculto en el enunciado de la metodología fabril y aún en la metodología académica.

Finalmente, profundizando sobre la emergencia de este nuevo actor trabajador, cabe preguntarse si resultará ésta una forma definitiva o sólo transicional, o bien incluso transitoria. En cuanto a la transición dependerá tanto de las fuerzas internas que se desplieguen como de la evolución del sistema político-económico. En este sentido las "pulsiones" magníficas que observamos a nivel global tienden a la expulsión de la categoría asalariado, desdibujando -precarizando sistemáticamente- la categoría de trabajador universal, formal y en relación de dependencia, que había caracterizado el modelo capitalista de postguerra (1945-1975). El nuevo actor laboral está signado, sin duda, por representaciones cruzadas, por un pasado fabril y la impronta neoliberal de dilución de la categoría asalariada como así la tendencia a la reducción del empleo de la fuerza de trabajo. Este proceso implica la extinción social en dos sentidos, por un lado con la ruptura del lazo dependiente-protector, la profundización - o el retroceso- a los inicios del siglo pasado con la compra del trabajo despojado del trabajador y por otro con la extinción del salario de sobrevivencia. La situación se extrema en este cruce y el cotrabajo emerge como la resistencia a la supresión; es así como se presentan las nuevas características en las nuevas luchas laborales encarnadas en este caso en las empresas recuperadas.

\section{A modo de epílogo, para recomenzar}

\section{Palabras de un Representante de la Empresa Recuperada I MPA luego de releer lo co-escrito:}

“... Estoy convencido de que todo debate sobre los cambios de las bases simbólicas referenciales es la clave del triunfo de las luchas futuras. En realidad lo ha sido siempre en la historia, pero la base tecnológica DEL EX CAPITALISMO que vivimos obligará a los actores sociales y a los intelectuales a una revolución simbólica sin antecedentes en torno al concepto del trabajo. 
No hay otra forma de resolver la problemática que significan los 84 millones por año de trabajadores en el mundo que todos los años firman su primera solicitud de empleo. En consecuencia este trabajo, lo interpreto como el primer paso con un gran potencial.

Se recuperan en el mismo conceptos como el de 'sana emulación' o conceptos análogos que actualizan y demuestran la vigencia del concepto de emulación, que el Che planteó como Ministro de Industrias para reemplazar la competencia liberal.

Desde el Centro Cultural y de nuestra militancia política venimos sosteniendo, que lo estratégico es la violencia semántica, sobre el capital simbólico del sistema que es obsoleto. Cuando leemos la frase de un compañero, rescatada por nuestra investigación que dice 'acá se trata de compañeros, ya no se trata de obreros', encierra un potencial de desarticulación que abarca desde el leninismo hasta el liberalismo, pasando por el poder sindical burocrático y las falsas divisiones entre sectores medios y trabajadores manuales. Esto demuestra para nosotros que la violencia semántica tiene una base material histórica y está en curso.

Otro aspecto, muy rescatable de la investigación, es lo referido a la disolución de las jerarquías, patrón-obrero, jefes-obreros. Creo que en esta reflexión falta, incorporar algo cualitativamente muy potente, derivado de esa disolución. Nos referimos a que esa disolución nace en lo interno de la fábrica y se extiende a las instituciones superestructurales del sistema. El Juez deja de ser un poder oculto y anónimo y ante el objetivo de recuperar la fuente de trabajo, los trabajadores en todos los casos de las empresas recuperadas, se ubican espiritualmente en relación de igualdad jurídica frente al Poder Judicial, obligándolo a las siguientes desestructuraciones:

1- Cambio en las rutinas burocráticas, sacando a los jueces del poder anónimo que ejercen a través de la fuerzas policiales y otras instituciones, que ejecutan sus órdenes: desalojos, remates, plazos para decretar quiebras, jerarquías entre los diversos derechos que amenazan destruir la fuente de trabajo, posibilidad de acuerdos parajudiciales con rematadores, síndicos y chatarreros, etc). El cambio de actitud de los trabajadores que pasaron de pretender simplemente cobrar su indemnización a organizarse para mantener funcionado o reabrir fuentes de trabajo, tiende a igualar la jerarquía entre todos esos actores, desestructurando el discurso del poder.

2- Cambio en los poderes legislativos, los trabajadores de las empresas recuperadas, hoy debaten de igual a igual y en muchos casos enseñan como cambiar las leyes en favor 
de los trabajadores, a los legisladores provinciales y nacionales, lo cual implica una disolución de la jerarquía entre los legisladores y el pueblo. Revirtiendo en esta práctica el obsoleto principio constitucional de que el pueblo no delibera sino es a través de sus representantes. Por ejemplo la Ley de Expropiaciones que no fue utilizada durante varias décadas en la Argentina fue desempolvada, puesta en vigencia y actualizada por las empresas recuperadas. Otro caso, la adopción de políticas activas de Estado iniciada por la ley de IMPA - Junio 2001 - de la legislatura de Buenos Aires, significó un recupero de dichas políticas, hacia la producción, cuando el paradigma de las políticas liberales con el Gobierno de la Alianza continuaba con los ejes de Estado implantados por la década menemista. Esa primera ley arrancada a fuerza de movilización, habilitó numerosas leyes posteriores. Otro caso, las empresas recuperadas antes de constituirse como movimiento, coordinaron acciones de movilización para que el Poder Legislativo Nacional, suspendiera la Ley de Quiebras, cosa que se logró y se mantuvo durante más de un año, obligando al FMI a una inusitada presión sobre el Poder Legislativo que fue denunciado a su vez por IMPA como delito de extorsión de un poder internacional sobre los poderes de la República. Lamentablemente 'los extorsionados legisladores' no asumieron la denuncia y no se animaron a constituirse en querellantes. Con lo cual en este caso ya no sólo hay disolución de las jerarquías entre los trabajadores y el Poder Legislativo, sino que se produjo un paso más, se invirtió la jerarquía en términos políticos al hacer las empresas recuperadas un planteo político superior al de los legisladores nacionales, que estos no se animaron a continuar.

3- Disolución frente a los poderes policiales. En el debate cara a cara en los diversos intentos de desalojo, los poderes policiales en la mayoría de los casos terminan reconociendo que los trabajadores tienen razón, y ellos solamente cumplen ordenes que les da el Juez. Frente a lo cual el discurso de las empresas recuperadas, es que no acepta una 'nueva obediencia debida y que la policía lo que tiene que hacer es pedirle al Juez que baje y dé la cara y las órdenes, después de discutir en una asamblea con los trabajadores. Esta práctica significó revertir decisiones judiciales, en los casos de Lavalan, Chilavert. etc.

Es dable reconocer que el único sector del poder del Estado, en que todavía las empresas recuperadas no lograron invertir la jerarquía es en todo lo referido a la normativa del sector financiero, hacia lo cual tienen políticas puntuales, pero a la fecha no han sido escuchadas.

Finalmente como última observación, de la investigación, surge el concepto del 'proceso de horizontalización' de decisiones, control y construcción. La potencialidad de ese concepto para explicar las causas de la crisis mundial y su solución, es incalculable. No ha 
sido aún desarrollado en lo más mínimo. La horizontalidad no es un concepto bondadoso post-moderno que nace por oposición a la verticalidad con que funcionaron las sociedades en el siglo XX. La horizontalidad es producto de un proceso material, económico hist órico, originado por los efectos de las diversas revoluciones tecnológicas de los últimos 20 años. Las empresas recuperadas son el fenómeno social de mayor potencial político de cara a la discusión de ese proceso tecnológico mundial que se sigue profundizando.

Esto no es, aunque pueda parecer, un mesianismo político, sino que las empresas recuperadas son una traba a la dinámica de extinción de las unidades productivas que SON TEORICAMENTE INEFICIENTES. ES LA CONTRADICCIÓN ENTRE LA INGENIERIA SOCIAL Y LA INGENIERIA TECNOLOGICA. En este sentido es de rescatar la reflexión de Peter Drucker en su último libro. Este científico de la Administración de Empresas, de Multinacionales con máximo prestigio en EE.UU. no es sospechable de intenciones subversivas. Sin embargo concluye con un pronóstico que en 10 años la tecnología no será un factor importante en los problemas de la sociedad y que el único problema, será el de las nuevas instituciones.

Las Empresas Recuperadas, con todas las implicaciones institucionales, ya descriptas, son un paso contundente en ese camino. Si la traba a la ubicación de los excedentes mundiales de producción, se extiende en la Argentina y en otros países, significará que los países centrales van a ser cada vez más afectados en su posibilidad de ubicar sus excedentes de producción industrial en los países periféricos. Este es el capital simbólico político de las empresas recuperadas que aún no ha sido planteado y que nos proponemos desarrollar en el año 2004. Es posible que por esta razón no haya respuestas del Poder Ejecutivo en el área financiera (como si lo ha habido en el área legislativa, judicial e incluso de los poderes represivos) a favor de las empresas recuperadas. Esta perspectiva, aplicada al concepto principal de la investigación, sintetizado en la expresión 'el co-trabajo emerge como la resistencia a la supresión del trabajo', es para nosotros el eje de las investigaciones futuras que propusimos al inicio de estas reflexiones".

\section{Notas}

1. Entendemos por "coproducción investigativa" a la relación que se establece entre el saber científico y otros saberes (en este caso, el saber de los trabajadores de IMPA), y que confluyen en un proceso de co-descubrimiento. Este proceso no se reduce a la pura reflexión individual sino a la producción (co-producción), a la investigación (coinvestigación), en el encuentro dialógico entre distintos actores y sus distintas perspectivas (conciliadas o confrontadas), que posibilitan la producción colectiva del conocimiento (Bialakowsky, Rosendo y Haimovici, 2002). 
2. Según Sautú (2003) el estudio de caso contempla tres características: 1. se focaliza sobre una situación, hecho o programa particular a ún cuando se constituya en un caso entre otros, con los cuales comparte ciertos rasgos; 2 . posee un alto contenido descriptivo que posibilita evidenciar las complejidades del caso; 3 . el estudio de caso posibilita encarar explicaciones en relación a cómo suceden los hechos y por qué, y cuáles las razones y el contexto en el que ocurren.

3. Se estima entre 120 y 130 el total de empresas recuperadas o en proceso de recuperación por sus trabajadores. Fuente: Vox Populi - Estudios Sociales (2003) En http://www.mner.org.ar/documentos.htm

4. La evolución de la tasa de desocupación en los 90 presenta altibajos que van del $8.6 \%$ a comienzos de los 90 , con picos del $18.4 \%$ en 1995 alcanzando $21.5 \%$ en mayo del 2002, como consecuencia de la profundización de la crisis sociopolítica desatada a fines del 2001. Fuente: INDEC: Cuadro de Devolución de las tasas de actividad, empleo, desocupación y subocupación. Total de aglomerados urbanos desde 1974 en adelante. En cuanto a la evolución del problema de la pobreza, para el caso del Gran Buenos Aires, la cantidad de personas por debajo de la línea de pobreza, proceso inflacionario mediante, era del $33.7 \%$ en octubre de 1990 , con una tendencia a disminuir hasta que en 1995 alcanza el 24, 8\%. La tendencia de este indicador a aumentar no logró revertirse, por el contrario hacia octubre de 2002 supera el 54\%. Asimismo, la línea de indigencia, pasó de 6.6\% en 1990 al 6.3\% en 1995, alcanzando en octubre de 2002 el 24.7\%. Fuente: INDEC (2002).

5. El coeficiente de Gini para 1991 era de 0.42 mientras que en mayo de 2002 alcanzaba 0.514 puntos. Fuente: CEDLAS.

6. La Ley de Quiebras dispone que una vez dictada tal medida, hay cuatro meses para rematar los bienes de la empresa, obtener el mejor precio posible y cancelar con lo recaudado las deudas. Si el juez demora esta liquidación, puede ser llevado a juicio por incumplimiento. [...] En el Movimiento Nacional de Empresas Recuperadas (MNER) proponen modificar dos artículos de la Ley de Quiebras. “El 217, que ordena rematar los bienes dentro de los cuatro meses, y el 211, para que los créditos de los trabajadores y del Estado (los impuestos adeudados) se puedan compensar traspasándoles las máquinas y el inmueble", dice el abogado Diego Kravetz (Citado por Vales).

7. Los objetivos enunciados por el MNER son: a) reclamar por la modificación de la Ley de Quiebras, que el trabajo sea considerado un bien social, toda unidad productiva que cierra debe ser puesta en manos de sus trabajadores; b) propiciar la creación de un Fondo Fiduciario que surja del aporte de las Empresas Recuperadas que se encuentren en mejor condición económica y de aportes del Estado Nacional para constituir capital de trabajo para las empresas recuperadas que comienzan a desarrollarse; c) desarrollar estrategias de integración vertical y horizontal potenciándose entre si, produciendo y apropiándose de los excedentes generados conformando cadenas altamente competitivas, incorporando tecnología moderna y adecuada con capacitación en la gesti ón; d) promover que los recursos que genera el MNER vuelvan al MNER, posibilitando la creación de una sociedad de garantías recíprocas integrada por las empresas del movimiento, cooperativas, mutuales, el Estado y bancos oficiales que quieran asociarse al emprendimiento; e) 
solicitar apoyo de los organismos de investigación, a las universidades y a los especialistas para definir con el mayor nivel de rigor científico los indicadores de eficiencia acordes a las actividades que desarrollan las empresas del MNER; f) constituir redes de consumidores, usuarios y pequeños comerciantes solidariamente organizados para defender sus derechos y promover pautas que favorezcan el consumo de bienes de producción nacional como forma de garantizar las fuentes de trabajo y como lucha contra el desempleo; g) estudiar variantes a los sistemas previsionales vigentes (Reparto y Capitalización) que garanticen una jubilación digna para los compañeros; h) conformar la Obra Social de los Trabajadores de las Empresas Recuperadas a partir de un criterio distinto al de la medicina mercantilista, haciendo hincapié en la prevención, optimizando los recursos y teniendo como objetivo brindar una cobertura completa y solidaria a los trabajadores y su grupo familiar (www.mner.org.ar).

8. En la Provincia de Buenos Aires, el Movimiento Nacional de Empresas Recuperadas (MNER) presentó en las últimas elecciones candidatos a diputado bonaerense, a vice-gobernador y a intendente a través del Polo Social. Mientras que en la Ciudad de Buenos Aires, las postulaciones se vehiculizaron a través del Partido de la Revolución Democrática (PRD), que lidera Miguel Bonasso. El abogado del movimiento, Diego Kravetz, quien encabezaba la lista de candidatos a la Legislatura ocupará desde diciembre de 2003 una banca. Por fuera del MNER, Celia Martínez, obrera de la textil Brukman, fue como candidata a diputada nacional del Partido de los Trabajadores Socialistas (PTS) y su compañero de trabajo Juan Carlos Ragghini, como candidato a legislador del Partido Obrero (PO). Daniel López, de Ghelco, integró la nómina de legisladores de Izquierda Unida (IU). (Murúa, 2003).

9. Según Dejours y otros (1998), una 'técnica' puede ser definida como un "acto tradicional eficaz". Eficaz, porque tiene un efecto detectable en el mundo, de lo contrario un acto no podría calificarse como técnica.

\section{Referencias Bibliográficas}

1. Antunes, Ricardo (1999), Adiós al trabajo? Ensayo sobre las metamorfosis y el rol central del mundo del trabajo, Ed. Antídoto, Buenos Aires.

2. Battistini, Osvaldo R. (2003), “La democracia constituida sobre la violencia”, en La atmósfera incandescente. Escritos políticos sobre la Argentina movilizada, $O$. Battistini (coord..), Asociación Trabajo y Sociedad, Buenos Aires.

3. Bauman, Zygmunt (1998), Modernidad y Holocausto, Ed. Sequitur, Toledo, España.

4. Bauman, Zygmunt (1999), En busca de la política, FCE, Buenos Aires.

5. Bauman, Zygmunt (2002) "Introducción", La cultura como praxis, Ed. Paidos, Buenos Aires. 
6. Bialakowsky, Alberto L. y Fernández, Beatriz (1994), Las articulaciones laborales.

Los estibadores del puerto de Buenos Aires, Centro Editor de América Latina, Buenos Aires.

7. Bialakowsky, Alberto et al (2003), “Conclusiones", Taller 51: “Las Nuevas Formas de Gestión y Autogestión de los Trabajadores", Compact Disk: V Jornadas de Sociología, Carrera de Sociología, Facultad de Ciencias Sociales, Universidad de Buenos Aires.

8. Bialakowsky, Alberto y Rosendo, Ernestina (1998), "Salud mental y flexibilización laboral: Una perspectiva antropológica" en Vertex. Revista Argentina de Psiquiatría, Volumen IX, No. 31, Buenos Aires.

9. Bialakowsky, Alberto; Rosendo, Ernestina; Haimovici, Nora (2002), “El encuentro de los discursos" en Revista de la Facultad de Ciencias Sociales, Facultad de Ciencias Sociales, UBA, No. 51, Buenos Aires.

10. Bourdieu, Pierre (1988), "Espacio social y poder simbólico" en Cosas Dichas, Gedisa, Bs. As.

11. Bourdieu, Pierre (1999), "Efectos de lugar" en La miseria del mundo, FCE, Buenos Aires.

12. Cafassi, Emilia (2002), Olla a presión, Libros del Rojas. Universidad de Buenos Aires, Buenos Aires.

13. Carpintero, Enrique y Hernández, Mario (comp.) (2002), Produciendo realidad. Las empresas comunitarias, Grissinopoli, Río Turbio, Zanón, Brukman, Gral. Moscón, Topía - La Maza, Buenos Aires.

14. Centro de Estudios Distributivos, Laborales y Sociales (CEDLAS). En. www.depeco. econo.unlp.edu.ar.

15. Colectivo Situaciones (2002), 19 y 20. Apuntes para el nuevo protagonismo social, Ediciones de Mano en Mano, Argentina.

16. Contrera, Elisabet (2003), Las quiebras dentro del Movimiento en http://www. redcom.org/ps2003/go3/mnerymefr.htm 
17. Dejours, C.; Dessors, D.; Molinier, P. (1998a), “Para comprender la resistencia al cambio" en Organización del trabajo y salud. De la Psicopatología a la Psicodinámica del trabajo, Lumen Hvmanitas, Argentina.

18. Dejours, Christophe (1998b), El factor humano, Lumen Hvmanitas, Argentina.

19. Dinerstein, Ana C. (2003), “El desempleo, espacio de subjetivización invisible y los piqueteros", en Herramienta, No. 22, Buenos Aires.

20. Escalera Reyes, Javier y Ruiz Ballesteros, Esteban (1996), “De ser obrero a ser empresario. S.A.P. para la viabilidad sociotécnica de Minas de Río Tinto (Sociedad Anónima Laboral)", Departamento Antropología Social, Sociología y Trabajo Social, Universidad de Sevilla, en VII Congreso de Antropología Social, Zaragoza. España.

21. Foucault, Michel (1978), "La Gubernamentalidad", en Espacios de Poder, M. Foucault y otros, Ed. La Piqueta (1991), Madrid.

22. Foucault, Michel (1984), “La ética del cuidado de uno mismo como práctica de la libertad", en Hermeneútica del sujeto, Altamira (1996), La Plata, Argentina.

23. Gambina, Julio C. (2003), Empresas Recuperadas en Argentina en http://www. elchenque.com. ar/eco/temrel/03/emprerecu.htm

24. Gaudemar, Jean Paul de (1978), “Preliminares para una genealogía de las formas de disciplina en el proceso capitalista de trabajo" en Espacios de poder, Autores varios, Ed. La Piqueta.

25. Gutiérrez, A.B. (1994), Pierre Bourdieu: Las prácticas sociales, CEAL, Buenos Aires.

26. Hardt, Michael y Negri, Antonio (2002), I mperio, Paidós, Buenos Aires.

27. Holloway, John (2002), Cambiar el mundo sin tomar el poder. El significado de la revolución hoy, Herramienta-Universidad Autónoma de Puebla, Buenos Aires.

28. Instituto Nacional de Estudios y Censo (INDEC) (2002), Encuesta Permanente de Hogares. Buenos Aires. 
29. Marx, Karl (1988a), Cap. VI. Inédito "Subsunción formal y subsunción real" en EI Capital, Ed. S. XXI, México D.F.

30. Marx, Karl (1988b), Cap. XI. “Cooperación” en El Capital, Ed. S. XXI, México D.F.

31. Marx, Karl (1988c), Cap. I. “La mercancía” en El Capital, Ed. S. XXI, M éxico D.F.

32. Marx, Karl (1993), “Trabajo enajenado” en Manuscritos: economía y filosofía. Ediciones Altaya. Barcelona.

33. Marx, Karl (1999), “El carácter fetichista de la mercancía y su secreto" en EI Capital, Tomo I/Vol.I, FCE, México.

34. Morin, Edgar (1998), “La auto-organización de las actividades vivientes" en EI Método. La vida de la vida, Ed. Cátedra, Madrid.

35. Movimiento Nacional de Empresas Recuperadas (MNER), Primer Encuentro Nacional de Incubadoras de Empresas Solidarias en http://www.mner.org.ar/ principal.htm

36. Murúa, Eduardo (2003), “Las empresas en quiebra deberían ser cedidas a sus trabajadores", sección EI País, Página 12, 8/9/2003.

37. Palomino, Héctor (2003), "El movimiento de trabajadores de empresas recuperadas", Revista Sociedad. Revista de la Facultad de Ciencias Sociales de la UBA, № 20/21, Ed. Manantial, Buenos Aires.

38. Roca i Girona, Jordi (1996), "Ideologías sobre el trabajo y satisfacción laboral”, en VI I Congreso de Antropología Social, Zaragoza, Instituto Aragonés de Antropología, Federación de Asociaciones de Antropología del Estado Español, Zaragoza.

39. Roca i Girona, Jordi (2001), “¿Antropólogos en la empresa? A propósito de la (mal) llamada cultura de la empresa", en Rev. Etnográfica, Vol. V (1), España.

40. Sautú, Ruth (2003), Todo es teoría. Objetivos y métodos de investigación, Lumiere, Buenos Aires.

41. Sennett, Richard (2000), “El pronombre peligroso. La comunidad como remedio para los males del trabajo", en La Corrosión del Carácter. Las consecuencias personales 
del trabajo en el nuevo capitalismo, Editorial Anagrama, Barcelona.

42. Touraine, Alain (1992), “La conciencia obrera" en Rojas, E. y Proietti, A. (comp.) Sociología del Trabajo, Ed. Centro Editor de América Latina. Buenos Aires.

43. Vales, Laura (2003), “La participación política de los movimientos de empresas recuperadas. Las fábricas recuperadas hacen política", Sección El País, Página 12, $7 / 9 / 2003$.

44. Vales, Laura. “Argentina: empleo. Reclamos de los movimientos de empresas recuperadas. Desde una quiebra a una esperanza" en http://www.sindicatomercosul. com. br/noticias. asp? Numero $=8816$ 